\title{
Binding Arguments and Hidden Variables
}

\author{
Jonathan Cohen and Samuel C. Rickless
}

In recent years, several philosophers have appealed to evidence about binding relations to show that various linguistic expressions are represented (at some level of linguistic representation) as having hidden variables (e.g., Stanley (2002), 368-369; Stanley and Szabó (2000), 243). In particular, the idea is that binding interactions between the relevant expressions and natural language quantifiers are best explained by the hypothesis that those expressions harbor hidden but bindable variables. Recently, however, Herman Cappelen and Ernie Lepore have rejected such binding arguments for the presence of hidden variables on the grounds that they overgeneralize - that, if sound, such arguments would establish the presence of hidden variables in all sorts of expressions where it is implausible that they exist (Cappelen and Lepore (2005), Cappelen and Lepore (2002)). ${ }^{1}$ In what follows we respond to Cappelen's and Lepore's attempted reductio by bringing out crucial disanalogies between cases where the binding argument is successful and cases where it is not. But we have a deeper purpose than merely to respond to Cappelen and Lepore: we think the attempted reductio goes wrong by not taking sufficiently seriously the nature of the binding relation that holds between quantifiers and arguments/variables, and that our criticism will serve to highlight the nature and importance of this relation.

\section{From Binding to Hidden Variables}

As we noted, the binding argument adduces facts about variable binding as evidence for the presence of hidden variables in the representation of linguistic expressions. For example, the thought is that we can reveal the presence of a hidden variable in 'it is sunny' in (1) by demonstrating binding interactions between that variable and the quantifier 'Everywhere Sally goes' in (1b):

(1) It is sunny. ${ }^{2}$

\footnotetext{
${ }^{1}$ Cappelen and Lepore object to the postulation of hidden indexical variables on the further grounds that these variables (i) are insufficient to demonstrate semantic context sensitivity (which is perhaps the main reason philosophers and linguists have been interested in such variables), (ii) are not bindable by anaphora in the way that ordinary indexicals are, (iii) don't guarantee certain a priori truths of the kind that are ordinarily guaranteed for indexicals, and (iv) predict the availability of interpretations that are, they claim, unavailable. We'll have nothing to say about these other arguments.

${ }^{2}$ For the sake of uniformity, we'll use 'it is sunny' rather than the contracted form 'it's
} 
(1b) Everywhere Sally goes, it is sunny.

$\left(1 b^{*}\right)$ For every place, $x$, if Sally goes to $\mathrm{x}$, then it is sunny at $\mathrm{x}$.

$\left(1^{*}\right)$ It is sunny at place $\mathrm{x}$.

The idea is supposed to be that the interpretation of 'it is sunny' in (1b) depends somehow on the quantifier 'everywhere'; in particular, the thought goes that this dependency can be explained by the hypothesis that there is a hidden variable associated with 'it is sunny' to be bound by the quantifier. Thus, (1b) receives the quasi-regimentation $\left(1 b^{*}\right)$. If so, and assuming 'it is sunny' is semantically unambiguous, there must be a hidden variable associated with the same expression in (1); hence, the proper quasi-regimentation for (1) is $\left(1^{*}\right)$.

\section{A Reductio of The Binding Argument?}

Cappelen and Lepore (2005) argue that the binding argument just considered generalizes inappropriately (74-76). For example, they imagine a confused mathematical anthropologist who attempts to summarize the data collected by her graduate student assistant, Sally, by uttering $(2 \mathrm{~b}) .^{3}$ Following the form of the binding argument we've already seen, they suggest that, if the correct representation of $(2 \mathrm{~b})$ is $\left(2 \mathrm{~b}^{*}\right)$, then we are led to the conclusion that the correct representation of $(2)$ is $\left(2^{*}\right)$ :

(2b) Everywhere Sally goes, $2+2=4$.

$\left(\mathbf{2} \mathbf{b}^{*}\right)$ For every place, $\mathrm{x}$, if Sally goes to $\mathrm{x}$, then $2+2=4$ at $\mathrm{x}$.

(2) $2+2=4$.

(2*) $2+2=4$ at place $\mathrm{x}$.

But this is unpalatable - it is about as uncontroversial as things get in this neck of the woods that (2) does not contain a hidden argument place for places.

Moreover, they point out that further instances of the binding argument can be used to argue for further hidden argument places in (2). For example, they suggest that insofar as (2c) is appropriately represented by $\left(2 \mathrm{c}^{*}\right)$, the same reasons that motivate treating $(2)$ as $\left(2^{*}\right)$ in the wake of $(2 \mathrm{~b})$ should now incline us to render $(2)$ as $\left(2^{* *}\right)$ :

(2c) Everywhere Sally goes, whenever she goes there, $2+2=4$.

$\left(2 c^{*}\right)$ For every place, $\mathrm{x}$, for every time, $\mathrm{y}$, if Sally goes to $\mathrm{x}$ at $\mathrm{y}$, then $2+2=4$ at $\mathrm{x}$, at $\mathrm{y}$.

sunny' throughout, even though the presentations in Cappelen and Lepore (2005) and Cappelen and Lepore (2002) switch freely between the two forms.

${ }^{3}$ We've changed the example from Cappelen's and Lepore's presentation to bypass irrelevant issues about the first person indexical 'I'. Nothing turns on this change. 
$\left(2^{* *}\right) 2+2=4$ at place $\mathrm{x}$, at time $\mathrm{y}$.

But if this is correct, then the same strategy could be used to argue that (2) harbors hidden argument places for what Sally is wearing, what Sally had for breakfast, and indefinitely many other parameters. ${ }^{4}$

We assume (with Cappelen and Lepore) that it is unacceptable to posit hidden argument places in (2) for place, time, what Sally is wearing, and the like. Consequently, if we are to preserve the case for representing (1) with hidden argument places, we need to say why the binding argument regarding that example is plausible but that regarding (2) is not.

\section{A Crucial Disanalogy}

It turns out, however, that it is not hard to spot what seems to us an extremely relevant disanalogy between the cases. To see this difference, notice that the way the binding argument works is by moving from data about binding to a conclusion about the presence of a hidden variable. And this means that it is crucial to the success of a putative instance of the binding argument that we accept the data about binding on which the instance depends. In particular, it is crucial that we accept the claim that there is indeed a variable in the embedded sentence that gets bound when it is put inside the scope of a quantifier.

For example, the argument for a hidden domain variable in (1) depends on starting with the assumption that $\left(1 b^{*}\right)$ is the right analysis of $(1 b)$, which means assuming that 'everywhere' in (1b) binds a variable in its scope. This assumption is, we think, quite plausible with respect to (1b). In fact, we don't see how to capture the truth-conditions of $(1 \mathrm{~b})$ without making it. E.g., it is only by making this assumption that we capture the fact that (1b) requires its being sunny in Paris when Sally is in Paris, its being sunny in London when Sally is in London, that it is not sufficient for the truth of (1b) that it be sunny in London when Sally is in Paris, and so forth.

In contrast, it doesn't seem at all obvious that $\left(2 b^{*}\right)$ is the right analysis of $(2 \mathrm{~b})$. What is unobvious here is the thought that $(2 \mathrm{~b})$ must be regimented

\footnotetext{
${ }^{4}$ Cappelen and Lepore mention the argument of Davidson (1967) against treating adverbial modification by increasing the adicity of verbs as inspiring their generalization of binding arguments to ever more parameters. We find this invocation of Davidson more than a little ironic. Davidson's proposal gathers its plausibility largely from its ability to preserve inferences such as the following as simple instances of conjunction reduction:

Jones buttered the toast slowly, deliberately, in the bathroom, with a knife, at midnight. Therefore, Jones buttered the toast.

However, in order to preserve this inference, Davidson's semantics must (and does) ensure that 'Jones buttered the toast' has the same semantic interpretation in premise and conclusion; and to ensure this, Davidson needs the semantic interpretation of 'buttered' (say) to be independent of the presence or absence of modifying material such as 'deliberately' or 'in the bathroom'. Thus, the success of Davidson's proposal about adverbial modification depends crucially on his keeping firmly in view the distinction between cases where there are semantically relevant dependency relations and cases where there are not - a distinction which, we shall argue below, Cappelen's and Lepore's attempted reductio of the binding argument turns on ignoring.
} 
by a representation according to which its embedded phrase ' $2+2=4$ ' contains a bound variable. ${ }^{5}$ Instead, it seems to us that the following is a perfectly good regimentation (in the sense that it adequately captures the truth conditions) of $(2 \mathrm{~b})$ :

$\left(\mathbf{2} \mathbf{b}^{* *}\right)$ For every place, $\mathrm{x}$, if Sally goes to $\mathrm{x}$, then $2+2=4$.

Of course, $\left(2 b^{* *}\right)$ involves quantification over places; but crucially, and unlike $\left(2 b^{*}\right)$, there is no variable bound by this quantification over places that appears in the part of the representation corresponding to the embedded sentence ' $2+2=4$ '.

What evidence is there for thinking that $(2 \mathrm{~b})$ should be understood in terms of $\left(2 b^{* *}\right)$ rather than $\left(2 b^{*}\right)$ ?

In general, if an embedded expression contains a hidden argument place bound by a quantifier, we should predict that removing the quantifier and replacing the variable by different names (i.e., assigning different values to the argument in that argument place) can have an effect on the interpretation of the embedded expression. For example, applying this manipulation to (1b) yields sentences such as the following:

(1bP) If Sally goes to Paris, then it is sunny (in Paris).

(1bL) If Sally goes to London, then it is sunny (in London).

And it seems clear that (1bP) and (1bL) differ in their semantic interpretation (e.g., they differ in their truth conditions).

In contrast, as far as we can see, the interpretation of the embedded sentence ' $2+2=4$ ' in $(2 \mathrm{~b})$ does not depend on which value is assigned to the alleged location variable. As one reflection of this fact, observe that the truth conditions of $(2 \mathrm{~b})$ are identical to those of $(2 \mathrm{bP})$ and $(2 \mathrm{bL})$ :

(2bP) If Sally goes to Paris, $2+2=4$ in Paris.

(2bL) If Sally goes to London, $2+2=4$ in London.

(Cappelen's and Lepore's confused mathematical anthropologist is ignorant of the fact that the interpretation of ' $2+2=4$ ' is independent of variable assignments; but that's exactly what makes her confused.)

But this observation drains the force of the proposed reductio. Since the binding argument for treating (1) by $\left(1^{*}\right)$ depends on finding a binding relation in (1b), and since there is no analogous evidence in favor of that sort of binding relation in $(2 \mathrm{~b})$, there is no reason to accept a version of the binding argument for treating $(2)$ by $\left(2^{*}\right)$.

\footnotetext{
${ }^{5}$ In saying this, we agree with Stanley (2005), who also denies that $\left(2 \mathrm{~b}^{*}\right)$ is the correct reading of $(2 \mathrm{~b})(244)$. But Stanley does not provide what he takes to be the correct reading of $(2 \mathrm{~b})$, and does not provide evidence for any alternative reading (nor does he draw a more general diagnosis about what Cappelen and Lepore have missed about the nature of binding, as we do in $§ 5$ ). This reduces his disagreement with Cappelen and Lepore on this issue to a stalemate. We hope to break the stalemate in Stanley's favor.
} 


\section{Objection and Reply}

One might object that appealing to the invariance in truth conditions discussed above is an imperfect test for differences in the interpretation of the contained phrase ' $2+2=4$ ', since the necessary truth of that phrase would also explain the invariability in the truth conditions of the sentences in which it is embedded. Likewise for this example, which we owe to Zoltan Szabó:

(3b) Everywhere Sally goes, she goes.

(3b*) For all places, $x$, if Sally goes to $x$, then Sally goes to $x$.

(3bP) If Sally goes to Paris, then Sally goes to Paris.

(3bL) If Sally goes to London, then Sally goes to London.

Here, again, the necessary truth of the embedded expression in (3b) provides an explanation of the identity in the truth-conditions of $(3 \mathrm{bP})$ and $(3 \mathrm{bL})-$ an explanation that can then be proposed as an alternative to the hypothesis that the interpretation of the embedded phrase in $(3 \mathrm{~b})$ is not dependent on the value assigned to an argument for places. (A similar point applies to examples that involve necessarily false embedded sentences.)

But this objection is ultimately unpersuasive. For Cappelen's and Lepore's choice to run their reductio on a necessary sentence (a choice on which, we suspect, much of the apparent force of their argument depends) invites us to misidentify fixity of truth value for fixity of semantic interpretation. When we avoid this confusion by restricting ourselves to instances of the binding argument involving contingent sentences, our test once again shows up a salient distinction between cases where the binding argument goes through and cases where it does not. Thus, consider:

(4) $E=m c^{2}$.

(4b) Everywhere Sally goes, $E=m c^{2}$.

(4b*) For all places, $x$, if Sally goes to $x, E=m c^{2}$ at $x$.

(4bP) If Sally goes to Paris, $E=m c^{2}$ in Paris.

(4bL) If Sally goes to London, $E=m c^{2}$ in London.

$\left(4 \mathbf{b}^{* *}\right)$ For all places, $x$, if Sally goes to $x, E=m c^{2}$.

Since the confounding issue of (metaphysical) necessity doesn't arise here, the identity of truth conditions under various assignments to the variable ' $x$ ' is good evidence for the semantic insensitivity of the consequent of $\left(4 b^{*}\right)$ to an alleged argument place for locations. This in turn is good evidence for the absence of such an argument place in (4b), which is to say that (4b) is better regimented as $\left(4 b^{* *}\right)$ than as $\left(4 b^{*}\right)$. 


\section{Moral: The Nature of Binding}

In our view, the difference between examples like (1b) on the one hand and (2b), $(3 \mathrm{~b})$, and $(4 \mathrm{~b})$ on the other - a difference fatal to Cappelen's and Lepore's attempted reductio - goes to the heart of what variable binding is about. Saying that a variable in an embedded sentence is bound by a quantifier amounts to saying that the interpretation of that embedded sentence is systematically affected by the quantifier. In particular, once unembedded, the interpretation of the previously embedded sentence depends systematically on the value taken by its variable. That 'it is sunny' in (1b) exhibits this systematic dependence is what leads us to posit a bound variable in that subsentential expression (as per $\left(1 b^{*}\right)$ ). There is no such dependence of the interpretation of ' $2+2=4$ ' in $(2 \mathrm{~b})$ (or of the interpretation of ' $E=m c^{2}$ ' in (4b)). Consequently, there is no reason to render $(2 \mathrm{~b})$ by $\left(2 \mathrm{~b}^{*}\right)\left(/(4 \mathrm{~b})\right.$ by $\left.\left(4 \mathrm{~b}^{*}\right)\right)$; something like $\left(2 \mathrm{~b}^{* *}\right)\left(/\left(4 \mathrm{~b}^{* *}\right)\right)$ is preferable.

We conclude that, whatever the other virtues or vices of the binding argument for hidden argument places in (1), the argument does not overgeneralize egregiously in the way considered. The suggestion that it does, we suggest, is based on a failure to appreciate the nature of the binding relation itself. ${ }^{6}$

$$
\begin{array}{r}
\text { Jonathan Cohen } \\
\text { University of California, San Diego } \\
\text { 9500 Gilman Drive } \\
\text { La Jolla, CA 92093-0119 } \\
\text { joncohen@aardvark.ucsd.edu } \\
\text { Samuel C. Rickless } \\
\text { University of California, San Diego } \\
\text { 9500 Gilman Drive } \\
\text { La Jolla, CA 92093-0119 } \\
\text { srickless@ucsd.edu }
\end{array}
$$

\section{References}

Cappelen, H., and E. Lepore (2002) "Indexicality, Binding, Anaphora, and $A$ Priori Truth," Analysis 62:4, 271-281.

Cappelen, H., and E. Lepore (2005) Insensitive Semantics: A Defense of Semantic Minimalism and Speech Act Pluaralism, Blackwell, Oxford.

Davidson, D. (1967) "The Logical Form of Action Sentences," in N. Rescher, ed., The Logic of Decision and Action, University of Pittsburgh Press, Pittsburgh, 81-95. Reprinted in Davidson (1980), 105-148.

Davidson, D. (1980) Essays on Action and Events, Clarendon Press, Oxford.

Stanley, J. (2002) "Nominal Restriction," in G. Preyer and G. Peter, eds., Logical Form and Language, Oxford University Press, Oxford, 365-388.

\footnotetext{
${ }^{6}$ We are grateful to Kent Bach, Delia Graff, Jason Stanley, Zoltan Szabó, Nellie Wieland, and an anonymous referee for this journal for helpful discussion of these matters.
} 
Stanley, J. (2005) "Semantics in Context," in G. Preyer and G. Peter, eds., Contextualism and Philosophy: Knowledge, Meaning, and Truth, Oxford University Press, Oxford, 221-253.

Stanley, J., and Z. Szabó (2000) "On Quantifier Domain Restriction," Mind and Language 15, 219-261. 